\title{
Who would be a candidate for bridge to recovery during prolonged mechanical left ventricular support in idiopathic dilated cardiomyopathy?
}

Goro Matsumiya, MD, Osamu Monta, MD, Norihide Fukushima, MD, Yoshiki Sawa, MD, Toshihiro Funatsu, MD, Kouichi Toda, MD, and Hikaru Matsuda, MD

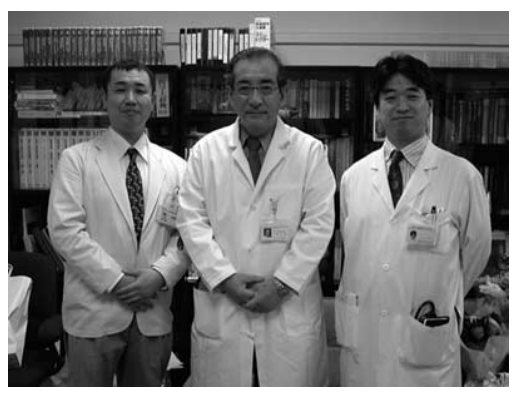

Monta, Matsudo, Matsumiya (left to right)
From the Division of Cardiovascular Surgery, Department of Surgery, Osaka University Graduate School of Medicine, Osaka, Japan.

Received for publication Dec 25, 2004; revisions received April 28, 2005; accepted for publication May 2, 2005.

Address for reprints: Goro Matsumiya, MD, 2-2 Yamada-oka, Suita, Osaka, 5650871, Japan (E-mail: matsumg@surg1. med.osaka-u.ac.jp).

J Thorac Cardiovasc Surg 2005;130:699-704 0022-5223/\$30.00

Copyright (C) 2005 by The American Association for Thoracic Surgery

doi:10.1016/j.jtcvs.2005.05.016
Objectives: We sought to elucidate the incidence and timing of myocardial recovery in patients with idiopathic dilated cardiomyopathy during prolonged support with a left ventricular assist system and to characterize the factors influencing this phenomenon.

Methods: Since 2000, 11 patients (mean age, 27.9 years) with end-stage heart failure caused by dilated cardiomyopathy were supported with a left ventricular assist system for more than 6 months. All patients had aggressive pharmacologic treatment, including $\beta$-blockers, together with unloading by means of a left ventricular assist system.

Results: During 239 to 663 days (mean, 453 days) of left ventricular assist system support, 5 patients had significant recovery of cardiac function and successfully underwent left ventricular assist system removal. All of these patients have remained in New York Heart Association class 1 during 8 to 29 months of follow-up. The improvement of left ventricular function started at various times. In particular, 2 of 5 recovered patients started to show improvement after 1 year of left ventricular assist system support. The myocardial fibrosis did not resolve but rather worsened in all patients during left ventricular assist system support. However, myocardial fibrosis was less severe in the recovered group $(17.7 \% \pm 8.2 \%$ at left ventricular assist system implantation vs $20.1 \% \pm 5.2 \%$ at explantation) in comparison with that in the nonrecovered group $(30.5 \% \pm 13.2 \%$ vs $48.4 \% \pm 5.1 \%)$ both before and after use of the left ventricular assist system.

Conclusion: Patients with severe congestive heart failure caused by dilated cardiomyopathy have a good chance of myocardial recovery and successful explantation of the left ventricular assist system. A relatively slow response of myocardial recovery during prolonged left ventricular assist system support was demonstrated in some patients. A lesser degree of fibrotic changes in the left ventricle was predictive of recovery.

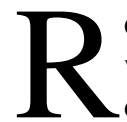
ecent reports have shown that idiopathic dilated cardiomyopathy (DCM), which had been considered irreversible, can be restored to almost normal cardiac function during support with a left ventricular assist system (LVAS), and recovery can be sustained for years after the explantation of the LVAS. ${ }^{1-3}$ Despite basic studies demonstrating the favorable effects of unloading of the left ventricle,${ }^{4-7}$ many questions still exist in clinical application of this strategy: Who can experience recovery and who cannot? What is the difference between these 2 groups? What percentage of patients have meaningful recovery? How long should one wait before abandoning the expectation of recovery? Past reports included patients with congestive heart failure (CHF) of many causes and those who were 
bridged to transplantation after a relatively short support period, ${ }^{8}$ and thus it has been hard to answer most of these questions.

In Japan we could restart heart transplantation in 1999 under new legislation. ${ }^{9}$ However, donor supply is still severely limited, and many patients require an LVAS while awaiting heart transplantation. This severe shortage of organ donors has resulted in a situation in which prolonged mechanical support exceeding 1 year is routinely required before heart transplantation. ${ }^{10}$ This gave us a unique opportunity to observe and evaluate the patients supported with an LVAS for a more prolonged period than in other countries. In this study we have analyzed the incidence and timing of myocardial recovery during this prolonged LVAS support and the factors influencing this phenomenon.

\section{Patients and Methods \\ Patients}

Ethical committee approval was obtained for this retrospective study, and informed consent was given by all the patients. From August 2000 to the end of 2003, a total of 13 patients with end-stage heart failure caused by DCM received the LVAS. The diagnosis of DCM based on histologic analyses, and no patient had signs of myocarditis. One patient died of massive cerebral bleeding at the second week from the operation, and another died of cerebral infarction at the third month. The other 11 patients survived for more than 6 months and underwent the serial evaluation of native cardiac function. Ten patients were male, and 1 patient was female. Age ranged from 15 to 38 years, with a mean of $27.9 \pm 7.6$ years. Preoperatively, all the patients had New York Heart Association class IV CHF symptoms and were dependent on inotropic support. All patients were taking angiotensin-converting enzyme inhibitors (ACEIs), diuretics, and spironolactone. $\beta$-Blockers were initiated in all patients, but 6 patients could not tolerate them because of hemodynamic deterioration or worsening of bronchial asthma. The indication for LVAS implantation was chronic progression of CHF in all the patients and not acute deterioration in stable CHF. Two patients required extracorporeal life support, 4 required an intraaortic balloon pump, and 5 were dependent on a ventilator. The history of heart failure ranged from 3 to 120 months, with a mean of $34 \pm 34.6$ months.

\section{Operations}

The type of device used was the HeartMate-IP (Thermo Cardiosystems, Inc, Woburn, Mass) in 8 patients, the HeartMate-VE in 2 patients, and the Novacor (Baxter Healthcare Corp, Novacor Div, Oakland, Calif) in 1 patient. The operation was performed as previously described. During cardiopulmonary bypass, apical coring and mitral annuloplasty (MAP), if indicated, were performed during cardioplegic cardiac arrest, and other procedures followed on the beating heart. MAP was performed to prevent recurrence of mitral regurgitation and to maintain left ventricular (LV) performance after possible LVAS removal. Simultaneous procedures performed during the operations were MAP with a 26-mm Cosgrove ring (Edwards Lifesciences, Irvine, Calif) for more than mild mitral regurgitation in 4 patients, tricuspid annuloplasty with the DeVega technique for more than mild tricuspid regurgitation in
2 patients, and implantation of a temporary right ventricular assist system in 1 patient.

\section{Heart Failure Treatment}

As soon as the patient's general condition was stabilized and organ dysfunction recovered, medical treatments for heart failure were initiated. This regimen included ACEIs, digoxin, spironolactone, and a $\beta$-blocker (carvedilol). Carvedilol was initiated at a dosage of $2.5 \mathrm{mg} / \mathrm{d}$ and doubled every week until a dosage of 20 to 40 $\mathrm{mg} / \mathrm{d}$ was reached. No patients were indicated for biventricular pacing. One patient had episodes of sustained ventricular tachycardia and received an automatic implantable cardioverter-defibrillator during LVAS support.

\section{Assessment of Cardiac Function and LVAS Weaning Protocol}

Echocardiography was performed every week in the first month and biweekly thereafter. Brain natriuretic peptide (BNP) levels were measured every week in the first month and monthly thereafter. After 3 months of entire unloading by LVAS, an LVAS off-test was performed in case echocardiography showed improvement of LV end-diastolic diameter of less than $55 \mathrm{~mm}$ and LV ejection fraction (LVEF) of more than $45 \%$ with a regularly running pump. The LVAS off-test was conducted as follows. During systemic heparinization $(3 \mathrm{mg} / \mathrm{kg}$ ), the LVAS pump rate was gradually decreased and stopped completely. After 10 minutes of heart beating without mechanical support, hemodynamic parameters and LV function were assessed by using a Swan-Ganz catheter and echocardiography. Then dobutamine was loaded up to $30 \mu \mathrm{g} \cdot \mathrm{kg}^{-1} \cdot \min ^{-1}$, and cardiac function was assessed. Weaning of LVAS was initiated in patients who had no deterioration of the hemodynamic and echocardiographic LV function during the LVAS halt and their further improvement with the increasing dose of dobutamine. The pump rate was set at the fixed mode and was decreased $10 \mathrm{pulses} / \mathrm{min}$ every week until the minimum pump rate of 50 pulses/min was reached. During this period, the BNP level was monitored every week. Unless the increase of BNP level occurred and the repeated LVAS off-test showed deterioration of LV function, explantation of the LVAS was performed.

\section{LVAS Explantation and Follow-up}

The LVAS was removed without cardiopulmonary bypass by using a modification of the method described by Hetzer and colleagues. ${ }^{11}$ In brief, a median skin incision over the pump pocket was extended toward the left anterior thoracotomy at the fifth or sixth intercostal space. The sternum was not split. During systemic heparinization, the pump was stopped, and the outflow and inflow conduits were clamped, cut, and oversewn. The apical cannula and most of the outflow graft were left in place. As soon as the endotracheal tube was extubated, heart failure medication was restarted. Cardiac function was assessed serially by means of echocardiography.

\section{Histologic Analyses}

Myocardial specimens were obtained at the time of LVAS implantation and then at the time of heart transplantation or autopsy. At LVAS explantation, myocardium was obtained with a biopsy needle from the anterior wall of the left ventricle. These specimens 
TABLE 1. Patients and follow-up data: Recovery group $(n=5)$

\begin{tabular}{lcccccc}
\hline & $\begin{array}{c}5 \\
\text { Days on } \\
\text { LVAS }\end{array}$ & $\begin{array}{c}\text { Recovery } \\
\text { course }\end{array}$ & $\begin{array}{c}\text { LVAs after } \\
\text { explantation }\end{array}$ & $\begin{array}{c}\text { LVEF* } \\
(\%)\end{array}$ & $\begin{array}{c}\text { LVDd* } \\
\text { (mm) }\end{array}$ & $\begin{array}{c}\text { BNP* } \\
\text { (pg/mL) }\end{array}$ \\
\hline $33 / \mathrm{M}$ & 460 & Early & 895 & 56 & 58 & 19.1 \\
$34 / \mathrm{M}$ & 435 & Early & 799 & 60 & 55 & 25.6 \\
$31 / \mathrm{M}$ & 239 & Early & 757 & 48 & 52 & 11.2 \\
20/M & 350 & Late & 554 & 53 & 51 & 53.6 \\
$15 / \mathrm{M}$ & 662 & Late & 248 & 54 & 50 & 32.4 \\
\hline
\end{tabular}

$\angle V A S$, Left ventricular assist system; $L V E F$, left ventricular ejection fraction; $L V D$, left ventricular diastolic dimension; $B N P$, brain natriuretic peptide.

*Data evaluated at the most recent outpatient clinic visit.

were fixed in $10 \%$ formalin, embedded in paraffin, and sectioned at a thickness of $5 \mu \mathrm{m}$. The specimens were stained with hematoxylin and eosin for light microscopic examination. The fibrosis area was calculated with the use of an image-analysis system (SPICCA II; Olympus, Tokyo, Japan), and the percentage of interstitial fibrosis was calculated as the average of the ratios of the fibrosis area to the total area from the 10 random sections.

\section{Statistical Analysis}

The quantitative data are presented as means \pm standard deviation. Comparisons among groups were calculated by using the MannWhitney $U$ test and the Fisher exact test for nonparametric data.

\section{Results}

The patients were categorized into the following 2 groups depending on their changes in cardiac function and subsequent clinical course (Tables 1 and 2). The recovery group includes the 5 patients who had significant recovery of cardiac function and underwent successful LVAS explantation after support periods of 239 to 662 days. They all are doing well and have been in New York Heart Association class 1 during 8 to 29 months of follow-up, with almost normal echocardiographic parameters and BNP levels. The nonrecovery group includes the 6 patients who had inade-

TABLE 2. Patients and follow-up data: Nonrecovery group $(n=6)$

\begin{tabular}{|c|c|c|c|}
\hline Age/sex & $\begin{array}{l}\text { Days on } \\
\text { LVAS }\end{array}$ & Prognosis & Cause of death \\
\hline $21 / F$ & 515 & $\begin{array}{l}\text { Heart } \\
\text { transplantation }\end{array}$ & \\
\hline $27 / M$ & 642 & Death & $\begin{array}{l}\text { Cerebral } \\
\text { infarction }\end{array}$ \\
\hline $37 / \mathrm{M}$ & 392 & Death & Infection \\
\hline $38 / \mathrm{M}$ & 244 & Death & Infection \\
\hline $30 / \mathrm{M}$ & 628 & Death & Infection \\
\hline $21 / \mathrm{M}$ & 395 & Death & Infection \\
\hline
\end{tabular}

LVAS, Left ventricular assist system. quate improvement of echocardiographic parameters during LVAS full support or hemodynamic deterioration or poor response to dobutamine stress during the LVAS off-test and could not undergo LVAS removal. Heart transplantation was performed after 515 days of LVAS support in 1 patient, and 5 patients died of infectious or cerebrovascular complications 244 to 642 days after the LVAS implantation.

Table 3 shows the characteristics of the patients before LVAS implantation in each group. There was no significant difference except in duration of heart failure symptoms before LVAS implantation, which was significantly longer in the nonrecovery group. $\beta$-Blocker therapy was tolerated only in $1(20 \%)$ patient of the recovery group compared with two thirds of the patients in the nonrecovery group, although this did not reach statistical significance. The changes of echocardiographic parameters after LVAS implantation were divided in the following 3 patterns (Figure 1). In all the nonrecovered patients, LVEF remained less than $40 \%$ throughout the course. The recovered patients were divided in 2 subgroups. Three patients had rapid improvement of LVEF in the first 3 months after LVAS

TABLE 3. Characteristics of patients

\begin{tabular}{|c|c|c|c|}
\hline & $\begin{array}{c}\text { Recovery } \\
\text { group }(n=5)\end{array}$ & $\begin{array}{c}\text { Nonrecovery } \\
\text { group }(n=5)\end{array}$ & $\begin{array}{c}P \\
\text { value }\end{array}$ \\
\hline Age (y) & $26.6 \pm 8.5$ & $29.0 \pm 7.4$ & NS \\
\hline Sex (M:F) & 5:0 & $5: 1$ & NS \\
\hline Duration of CHF (mo) & $10.2 \pm 8.0$ & $53.8 \pm 36.2$ & .028 \\
\hline $\begin{array}{l}\beta \text {-Blocker } \\
\quad \text { intolerance }\end{array}$ & $4 / 5(80 \%)$ & $2 / 6(33.3 \%)$ & NS \\
\hline LVEF (\%) & $17.4 \pm 4.8$ & $15.8 \pm 5.4$ & NS \\
\hline LVDd (mm) & $71.4 \pm 5.7$ & $73.3 \pm 7.2$ & NS \\
\hline $\mathrm{Cl}\left(\mathrm{L} \cdot \mathrm{min}^{-1} \cdot \mathrm{m}^{-2}\right)$ & $1.99 \pm 0.6$ & $1.60 \pm 0.5$ & NS \\
\hline MPAP (mm Hg) & $38.2 \pm 4.9$ & $39.7 \pm 10.7$ & NS \\
\hline \multicolumn{4}{|l|}{ Preoperative support } \\
\hline Inotropes & $5 / 5(100 \%)$ & $6 / 6(100 \%)$ & NS \\
\hline Ventilator & $3 / 5(60 \%)$ & $2 / 6(33.3 \%)$ & NS \\
\hline IABP & $3 / 5(60 \%)$ & $1 / 6(16.7 \%)$ & NS \\
\hline ECLS & $1 / 5(20 \%)$ & $1 / 6(16.7 \%)$ & NS \\
\hline \multicolumn{4}{|l|}{$\begin{array}{l}\text { Concomitant } \\
\text { procedure }\end{array}$} \\
\hline MAP & $1 / 5(20 \%)$ & $2 / 6(33.3 \%)$ & NS \\
\hline TAP & $0 / 5(0 \%)$ & $1 / 6(16.6 \%)$ & NS \\
\hline RVAS & $0 / 5(0 \%)$ & $1 / 6(16.6 \%)$ & NS \\
\hline LVAS support (d) & $429.4 \pm 156.5$ & $470.3 \pm 155.8$ & NS \\
\hline \multicolumn{4}{|l|}{ LVAS off-test* } \\
\hline MPAP (mm Hg) & $15.6 \pm 4.72$ & $33.5 \pm 14.8$ & NS \\
\hline $\mathrm{CI}\left(\mathrm{L} \cdot \mathrm{min}^{-1} \cdot \mathrm{m}^{-2}\right)$ & $3.27 \pm 0.44$ & $2.11 \pm 0.22$ & NS \\
\hline
\end{tabular}

$\overline{C H F}$, Congestive heart failure; $L V E F$, left ventricular ejection fraction; $L V D d$, left ventricular diastolic dimension; $\mathrm{Cl}$, cardiac index; MPAP, mean pulmonary artery pressure; $I A B P$, intra-aortic balloon pump; $E C L S$, extracorporeal life support; $M A P$, mitral annuloplasty; $T A P$, tricuspid annuloplasty; RVAS, right ventricular assist system; LVAS, left ventricular assist system; $N S$, not significant. *Measured with a Swan-Ganz catheter while the LVAS was turned off in 5 recovery and 3 nonrecovery group patients. 


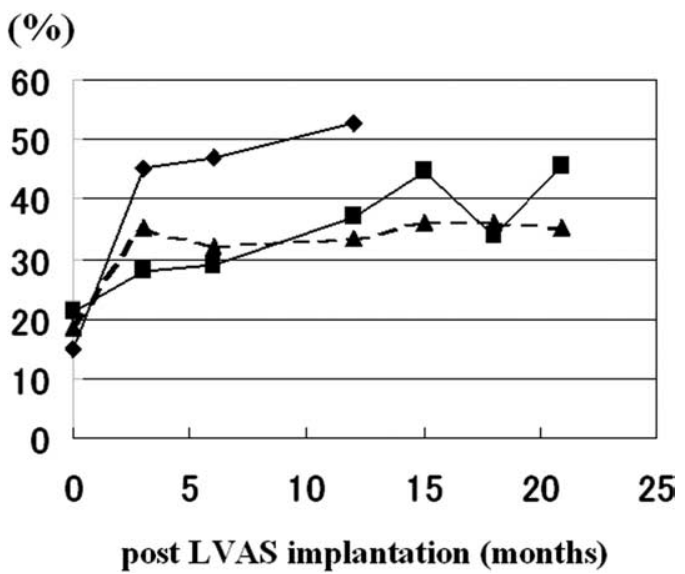

Figure 1. Change of LVEF. Changes of echocardiographic parameters after LVAS implantation were divided in 3 patterns. In all the nonrecovered patients, LVEF remained less than $\mathbf{4 0} \%$ throughout the course. Three patients had rapid improvement of LVEF in the first 3 months after LVAS implantation (early recovery group). Two patients had gradual improvement after 1 year and eventually reached the criteria for LVAS removal (late recovery group). Diamonds, Early recovery $(n=3)$; squares, late recovery $(n=2)$; triangles, nonrecovery $(n=6)$.

implantation (early recovery group). On the contrary, 2 patients had almost the same LVEF as the nonrecovered group for 1 year, but gradual improvement took place afterward and eventually reached the criteria for LVAS removal (late recovery group). The change in serum BNP level followed the reverse pattern of the change in LVEF in each group (Figure 2). In the 3 patients of the early recovery group, serum BNP level demonstrated a rapid decrease to the normal value in the first 3 months. The other 8 patients did not reach the normal value in 1 year. Although the nonrecovery group had an increase of BNP after 1 year, the other 2 patients in the late recovery group demonstrated a decrease to the normal value after 1 year. Histologically, the percentage of fibrosis at the time of LVAS implantation ranged between $8.1 \%$ and $51.6 \%$. The patients in the recovered group had significantly lower percentages of fibrosis $(17.7 \% \pm 8.2 \%)$ than those in the nonrecovered group $(30.5 \% \pm 13.2 \%)$. When we look at the change of fibrosis during LVAS support, myocardial fibrosis progressed in all the patients, including those in the recovery group. However, the percentage of fibrosis at LVAS explantation worsened more significantly in the nonrecovered group $(48.4 \%$ $\pm 5.1 \%)$ than in the recovered group $(20.1 \% \pm 5.2 \%$, Figure 3).

\section{Discussion}

Reverse remodeling of a failed left ventricle by means of mechanical unloading with LVAS is attracting increasing

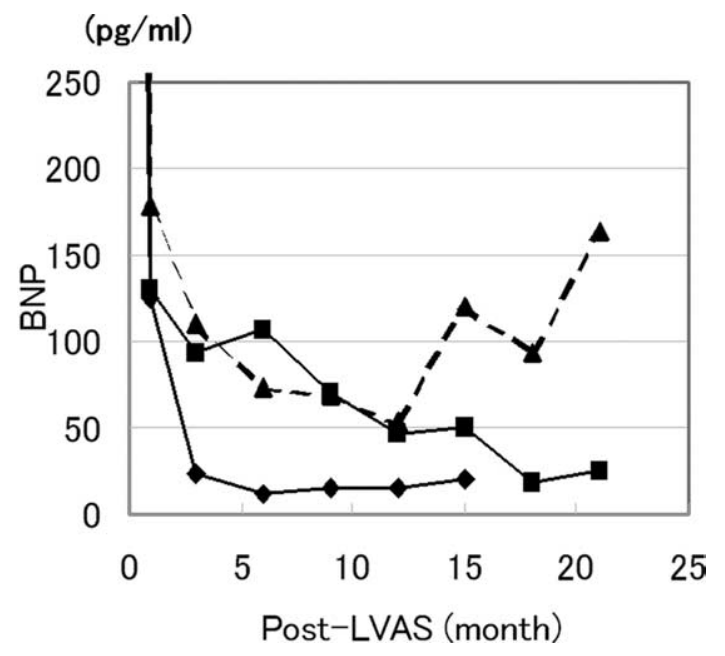

Figure 2. Change of BNP. The change of serum BNP level followed the reverse pattern of the change in LVEF. In the early recovery group serum BNP level demonstrated rapid decrease to the normal level in the first 3 months. Although the nonrecovery group had an increase of BNP after 1 year, the 2 patients in the late recovery group demonstrated a decrease to the normal level after 1 year. Diamonds, Early recovery $(\mathrm{n}=3)$; squares, late recovery $(n=2)$; triangles, nonrecovery $(n=6)$.

interest. ${ }^{12}$ However, many questions remain, especially regarding its clinical appearance and the mechanisms involved at the cell and tissue levels. ${ }^{13}$ Although the clinical observations vary in each report, relatively common find-

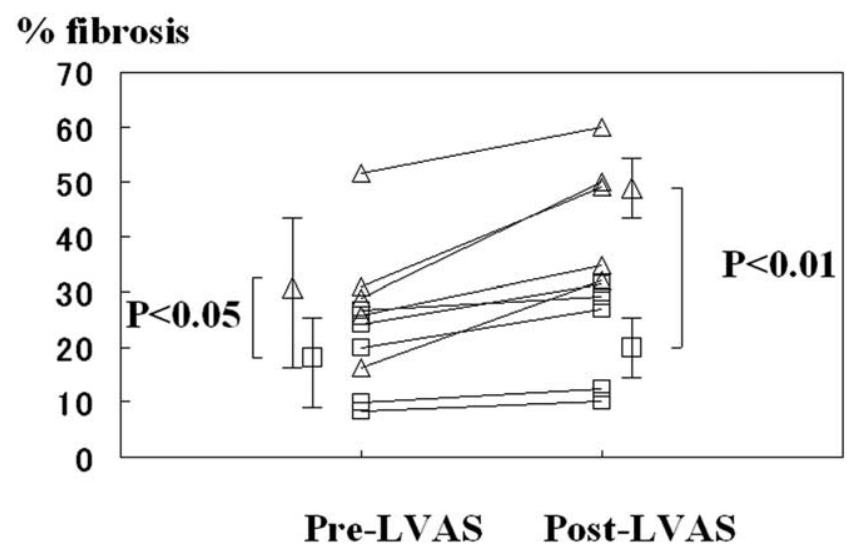

Figure 3. Change of myocardial fibrosis during LVAS support. The recovered patients had a significantly lower percentage of fibrosis $(17.7 \% \pm 8.2 \%)$ than the nonrecovered group $(30.5 \% \pm 13.2 \%)$ at LVAS implantation. In all patients, including the recovery group, myocardial fibrosis progressed during LVAS support. The percentage of fibrosis at the time of LVAS explantation was significantly higher in the nonrecovered group $(48.4 \% \pm 5.1 \%)$ than in the recovered group $(20.1 \% \pm 5.2 \%)$. Squares, Recovered; triangles, nonrecovered. 
ings are that recovery more likely occurs in the patients clinically characterized by younger age, shorter history of CHF, and application of the apical drainage type of LVAS. ${ }^{11}$ However, the incidence of myocardial recovery during LVAS support is variously reported to be between $4 \%^{8}$ and $33 \% .^{3}$ This discrepancy might have arisen from many issues. The patient population differed, and aggressiveness of pharmacologic treatment during LVAS support and its regimen, such as the use of $\beta_{1}$-blocker ${ }^{14}$ or $\beta_{2^{-}}$ agonist, ${ }^{3}$ varied. More importantly, the duration of LVAS support was significantly different because the bridge-totransplantation cases were included in most of the past reports. The time course of recovery of $\mathrm{LV}$ function has also not been clearly understood. Several reports have described that the early improvement of cardiac function is one of the predicting factors for successful recovery. Müller and associates ${ }^{1}$ showed that the LV end-diastolic diameter and LVEF 2 months after LVAS implantation significantly correlated with the sustained LV recovery. Sodian and coworkers ${ }^{15}$ demonstrated that BNP levels at the first week after LVAS implantation were significantly lower in the recovered patients. These observations partly coincide with our experience, in which 3 of 5 patients had quick recovery of LV function in the first 3 months. However, in 2 of 5 recovered patients, the significant recovery of LV function started after more than 1 year of support. This observation has been unique in our study. Hetzer and colleagues ${ }^{11}$ also described 2 patients who were weaned from LVAS after more than a year out of 23 patients who underwent LVAS explantation. However, those patients had marginal LV function before the explantation, had recurrence of CHF, and eventually required heart transplantation. They were clearly different from our patients, who had complete and sustained recovery. The mechanism of this late recovery is presently unknown. A prospective study with ACEIs and $\beta$-blockers demonstrated that the reverse remodeling process continues during 1 year of medical treatment. ${ }^{16}$ The multifactorial nature of the remodeling process might cause those time course differences in each patient. ${ }^{17}$ LVAS dysfunction negatively affected recovery in 2 patients, who had late occurrence of inflow valve regurgitation. Right heart function might also influence the recovery, but no patients in the present study had signs of right heart failure.

The other significant finding in our study is the important role of myocardial fibrosis in the functional recovery. Bruckner and associates ${ }^{18}$ demonstrated significant correlation between a lesser degree of preexisting fibrosis and greater improvements of cardiac function during LVAS support. Our study also showed a lesser degree of preoperative fibrotic change in the recovered ventricle. From our data, less than $30 \%$ of myocardial fibrosis predicts a good chance of successful weaning from the LVAS. It might be a reasonable explanation for this phenomenon that the re- covered patients deteriorated faster and reached the point when they required LVAS implantation sooner, and as a result, the fibrotic change did not progress to the point at which recovery is impossible.

There are conflicting reports on whether fibrosis progresses or regresses during LVAS support. Hetzer and colleagues ${ }^{14}$ demonstrated improvement of myocardial fibrosis during LVAS support in the recovered patients. On the contrary, there are several reports showing that unloading by the LVAS does not change preexisting fibrotic change. ${ }^{19}$ Our histologic analyses showed that the degree of fibrosis did not regress but rather worsened in most cases, including in 5 recovered patients, although the progression was much slower in the recovery group. A possible explanation for this discrepancy can be derived from the differences in LVAS duration, pharmacologic treatment, and aggressiveness of the weaning protocol. $\mathrm{Li}$ and associates ${ }^{5}$ showed no change of total collagen content, but reduced collagen damage manifested as the increased ratio of undenatured collagen during LVAS. Not only collagen content but also its quality can influence the LV function.

Why does cardiac function improve during LVAS support? There are reports demonstrating improvement of neurohormonal status, ${ }^{20}$ calcium handling in the sarcoplasmic reticulum, ${ }^{21}$ and myocardial response to adrenergic stimulus ${ }^{6}$ during LVAS support by hemodynamic normalization and unloading of the left ventricle. Sympathetic nerve overstimulation has one of the most significant correlations with the progression of CHF. $\beta$-Blocker therapy is now one of the most reliable modalities for heart failure treatment. Some of our patients, especially in the recovered group, were in severe heart failure and could not tolerate $\beta$-blocker therapy preoperatively. The LVAS enabled $\beta$-blocker therapy in all the patients, which might have contributed to the improvement of the sympathetic nerve function. We previously reported that the LVAS alone did not improve the sympathetic nerve activity, as assessed on the basis of iodine 125-labeled metaiodobenzylguanidine. ${ }^{22}$ The recovered patient in the present series showed serial improvement of the sympathetic nerve activity assessed by means of iodine 125-labeled metaiodobenzylguanidine (data not shown). The theoretic advantages of use of clenbuterol, a selective $\beta_{2}$-agonist, together with a $\beta_{1}$-antagonist have been described in several articles. It might become a useful adjunctive treatment to hasten recovery, although a larger multi-institutional study would be needed before its wider application.

\section{Clinical Implications}

Our data showed that in selected patients with DCM, weaning from the LVAS is a possible option. This phenomenon might occur more frequently than we previously expected, especially when the patients are supported for a prolonged 
period and meticulous heart failure treatment and careful evaluation are used. If we can determine those patients at an early stage after implantation, we can save the unnecessary heart transplantation and thus use the limited supply of donor hearts more effectively. From our experience and other recent reports, the degree of myocardial fibrosis is one of the key factors for predicting successful weaning from the LVAS. If a patient with DCM has less than $30 \%$ of fibrosis in the LV apex, possible recovery can be observed. If a more reliably used long-term device becomes available in the near future, those patients can be observed for at least 6 months to 1 year and then evaluated for recovery or transplantation candidacy.

\section{Limitations}

There are several limitations to this study. First, the number of the patients included in the study is small, and therefore selection bias is possible. In particular, our patient population was younger than that seen in past reports. The type of device might be another factor influencing myocardial recovery because the unloading effect is different in each device. All of our patients supported for a prolonged period received implantable pulsatile devices, which can achieve powerful unloading. In addition, ischemic cardiomyopathy was not included, and some of the patients had a relatively short history of CHF. These might have influenced the relatively high incidence of recovery in our patients. Our histologic analysis was performed with a myocardial specimen from the LV apex. Although there is a concern of regional heterogeneity of fibrotic change, most of the past studies have also used myocardium from the same area, which is the only available sample in the pre-LVAS situation.

\section{Conclusions}

Patients with severe CHF caused by DCM have a good chance of myocardial recovery and successful explantation of the LVAS. A relatively slow response of myocardial recovery during prolonged LVAS support was demonstrated in some patients. A lesser degree of fibrotic changes in the left ventricle was predictive of recovery.

\section{References}

1. Müller J, Wallukat G, Weng Y-G, et al. Weaning from mechanical cardiac support in patients with idiopathic dilated cardiomyopathy. Circulation. 1997;96:542-9.

2. Frazier $\mathrm{OH}$, Myers TJ. Left ventricular assist system as a bridge to myocardial recovery. Ann Thorac Surg. 1999;68:734-41.

3. Yacoub MH. A novel strategy to maximize the efficacy of left ventricular assist devices as a bridge to recovery. Eur Heart J. 2001;22: 534-40.

4. Bartling B, Milting H, Schumann H, et al. Myocardial gene expression of regulators of myocyte apoptosis and myocyte calcium homeostasis during hemodynamic unloading by ventricular assist devices in patients with end-stage heart failure. Circulation. 1999;100(suppl II):II216-23.

5. Li YY, Feng Y, McTiernan CF, et al. Downregulation of matrix metalloproteinases and reduction in collagen damage in the failing human heart after support with left ventricular assist devices. Circulation. 2001;104:1147-52.

6. Ogletree-Hughes ML, Stull LB, Sweet WE, Smedira NG, McCarthy $\mathrm{PM}$, Movarec CS. Mechanical unloading restores $\beta$-adrenergic responsiveness and reverses receptor downregulation in the failing human heart. Circulation. 2001;104:881-6.

7. Terracciano CMN, Harding SE, Adamson D, et al. Changes in sarcolemmal Ca entry and sarcoplasmic reticulum Ca content in ventricular myocytes from patients with end-stage heart failure following myocardial recovery after combined pharmacological and ventricular assist device therapy. Eur Heart J. 2003;24:1329-39.

8. Mancini DM, Beniaminovitz A, Levin $\mathrm{H}$, et al. Low incidence of myocardial recovery after left ventricular assist device implantation in patients with chronic heart failure. Circulation. 1998;98:2383-9.

9. Matsuda H, Fukushima N, Sawa Y, Nishimura M, Matsumiya G, Shirakura R. First brain dead donor heart transplantation under new legislation in Japan. Jpn J Thorac Cardiovasc Surg. 1999;47:499-505.

10. Matsuda H, Matsumiya G. Current status of left ventricular assist devices: the role in bridge to heart transplantation and future perspectives. J Artif Organs. 2003;6:157-61.

11. Hetzer R, Muller J, Weng Y-G, et al. Midterm follow-up of patients who underwent removal of a left ventricular assist device after cardiac recovery from end-stage dilated cardiomyopathy. J Thorac Cardiovasc Surg. 2000;120:843-55.

12. Margulies KB. Reversal mechanisms of left ventricular remodeling: lessons from left ventricular assist device experiments. J Card Fail. 2002;8(suppl):S500-5.

13. Reinlib L, Abraham W. Recovery from heart failure with circulatory assist: a working group of the national, heart, lung, and blood institute. J Card Fail. 2003;9:459-63.

14. Hetzer R, Müller JH, Weng Y-G, Loebe M, Wallukat G. Bridging-torecovery. Ann Thorac Surg. 2001;71(suppl):S109-13.

15. Sodian R, Loebe M, Schmitt C, et al. Decreased plasma concentration of brain natriuretic peptide as a potential indicator of cardiac recovery in patients supported by mechanical circulatory assist systems. $J$ Am Coll Cardiol. 2001;38:1942-9.

16. Doughty RN, Whalley GA, Gamble G, MacMahon S, Sharpe N. Left ventricular remodeling with carvedilol in patients with congestive heart failure due to ischemic heart disease. J Am Coll Cardiol. 1997; 29:1060-6

17. Cohn JN, Ferrari R, Sharpe N. Cardiac remodeling-concepts and clinical implications: a consensus paper from an international forum on cardiac remodeling. J Am Coll Cardiol. 2000;35:569-82.

18. Bruckner BA, Razeghi P, Stetson S, et al. Degree of cardiac fibrosis and hypertrophy at time of implantation predicts myocardial improvement during left ventricular assist device support. J Heart Lung Transplant. 2004;23:36-42.

19. Milting H, Jacob M, Kassner A, et al. The structural examination of myocardial samples from patients with end-stage heart failure supported by ventricular assist devices using electron microscopy and amino acid analysis reveals low degree of reverse remodeling. J Heart Lung Transplant. 2004;23:396-404.

20. James KB, McCarthy PM, Thomas JD, et al. Effect of the implantable left ventricular assist device on neuroendocrine activation in heart failure. Circulation. 1995;92(suppl II):II191-5.

21. Terracciano CMN, Hardy J, Birks EJ, Khaghani A, Banner NR, Yacoub $\mathrm{MH}$. Clinical recovery from end-stage heart failure using left-ventricular assist device and pharmacological therapy correlates with increased sarcoplasmic reticulum calcium content but not with regression of cellular hypertrophy. Circulation. 2004;109:2263-5.

22. Miyagawa S, Sawa Y, Fukushima N, et al. Analysis of sympathetic nerve activity in end-stage cardiomyopathy patients receiving left ventricular support. J Heart Lung Transplant. 2001;20:1181-7. 